\title{
Assessing Drug Use Indicators in Health Insurance Facilities, Gezira State, Sudan, 2017-2018
}

\author{
Sara Abdelrahman Ahmed1, Elnazeer Ibrahim Hamedelniel², Abubakr Khidir Yousif ${ }^{*}$ (]) \\ ${ }^{1}$ Medical Service Directorate, National Health Insurance Fund, Gezira, Sudan \\ ${ }^{2}$ Department of Pharmaceutics, College of Pharmacy, Omdurman Islamic University, Omdurman, Sudan \\ ${ }^{3} \mathrm{MPH}, \mathrm{MHPE}$, National Health Insurance Fund, Gezira, Sudan \\ Email: saraomer386@gmail.com, nizophar@hotmail.com, *abubarkhidir@hotmail.com
}

How to cite this paper: Ahmed, S.A., Hamedelniel, E.I. and Yousif, A.K. (2021) Assessing Drug Use Indicators in Health Insurance Facilities, Gezira State, Sudan, 2017-2018. Pharmacology \& Pharmacy, 12, 237-246. https://doi.org/10.4236/pp.2021.1210020

Received: August 24, 2021

Accepted: October 23, 2021

Published: October 26, 2021

Copyright ( 2021 by author(s) and Scientific Research Publishing Inc. This work is licensed under the Creative Commons Attribution International License (CC BY 4.0).

http://creativecommons.org/licenses/by/4.0/ (c) (i) Open Access

\begin{abstract}
Background: Inappropriate use of medicines is a global concern with serious consequences related to prescribing, dispensing, and use. WHO estimated that $50 \%$ of medicines are not used correctly on their journey from the facility to home. Objective: To assess medicines use using WHO drug core indicators regarding prescribing, patient, and facilities. Setting: Outpatients, Health centers in Wadmadani locality (Urban area) in Gezira State, Sudan. Method: A cross-sectional, prospective, analytical study was conducted in 30 health centers and 60 patients from each center were selected using a simple random sampling technique. WHO indicators form was used to collect data containing different variables. T-test at a level of confidence of $95 \%$ was used to test differences between indicators. Statistical Package for Social Science (SPSS) was used for data analysis. Results: The main prescribing indicators were 2.5 \pm 0.6 for drugs per encounter, $44.1 \% \pm 14.2 \%$. Generic $54 \pm 18.0$ antibiotics, $12.0 \% \pm 9.3 \%$ injectable, and $95.2 \% \pm 11.5 \%$ of drugs were prescribed according to the NHIF-EML. The main patient's indicators were, $2.9 \pm 0.8 \mathrm{mi}-$ nutes for consultation time, $99.5 \pm 36.8$ seconds for dispensing time, and $72.5 \% \pm 16.0 \%$ for medicines actually dispensed, $49.0 \% \pm 18.0 \%$ for medicines adequately labeled, and $22.5 \% \pm 7.3 \%$ of the patient's knowledge about the correct dose. The Facility specific indicators were $66.7 \%$ for the availability of a copy of EML, while the percentage of key drugs in the stock was $75.3 \%$ $\pm 11.6 \%$. No statistically significant differences were found between direct and indirect facilities except in generic prescribing. Main Outcome Measure: - Interventions to improve Generic and antibiotics prescribing indicators. • The patient-to-physician ratio should be revised to optimize consultation time. - The availability of key drugs should be improved to make sure effec-
\end{abstract}


tive treatment. - The pharmacy cadre should be oriented and trained to improve patients' compliance. Conclusion: The study concluded that there was irrational use of medicines when investigated by WHO drug core indicators. So, the study recommended interventions to improve the rationale prescribing, dispensing, and use of medicines.

\section{Keywords}

Assessing, Drug Use Indicators, National Health Insurance Fund, Gezira, Sudan

\section{Introduction}

Medicines play a crucial role in improving, restoring, and maintaining health. Availability, as well as the appropriate use of medicines, had become a global concern to achieve the desired outcomes [1]. The rational drug use as defined by the World Health Organization (WHO) "patients receive medications appropriate to their clinical needs, in doses that meet their individual requirements, for an adequate period of time, and at the lowest cost to them, and their communities" [2]. Unfortunately, more than $50 \%$ of all medicines are prescribed, dispensed, or used inappropriately [3]. The common problems of inappropriate use of medicines include polypharmacy (use of too many medicines/prescription), overuse of antibiotics and injections, failure to prescribe according to clinical guidelines, and poor patient adherence and compliance [3]. The dramatic increase in medicines expenditure due to irrational use can affect the quality of medical services delivered by the national health insurance fund (NHIF). The NHIF Gezira state served more than two million people in 2018 [4].

The NHIF provides a benefits package including all levels of healthcare providers (from medical assistants up to consultants). Broad medical care of inpatient and outpatient with all laboratory investigations, and diagnostic imaging, were delivered to insured patients. The insured patient pays only $25 \%$ of the cost of the medicines within the NHIF list. NHIF Gezira state provides medical services through 435 health centers and hospitals.

NHIF Essential Medicines List (NHIF-EML) version six, last updated in 2018, contained more than 600 generic items. The State Ministry of Health (SMOH) formulated a National Standard Treatment Guidelines (STG) for major clinical conditions, but adherence is not seen in practice. Antibiotics were often dispensed over the counter without a prescription. In order to improve rationale practice, the NHIF regulates and classified the medicine list according to the above-mentioned healthcare provider's levels.

The total pharmaceutical expenditure had been increased dramatically in the few past years. In the year 2018, it was reported more than $57 \%$ (the ideal $\leq 40 \%$ as stated in the NHIF indicators manual). Comparing with premium, medicines 
are determinant factors to NHIF to be live or destroyed. Improving dispensing and prescribing practice will improve health outcomes, reduce the cost, and improve the quality of services. Most literature that used WHO drug core indicators were descriptive and suggested analytical test was scarce. So this study used inferential statistics tests, which considered being novel and innovative in this area, for proper general interventions.

\subsection{Aim of the Study}

- Generally to investigate drug core indicators using WHO standard [2].

- Specifically to:

- Identify the extent of poly-pharmacy at health care facilities.

- Check the generic prescribing of medicines.

- Determine prescription patterns of antibiotics and injectable medicines.

- Measure the percentage of prescribed medicines with Essential medicines list.

- Estimate the consultation and dispensing time.

- Measure the percentage of medicines dispensed and adequately labeled.

- Evaluate the patient knowledge about their medicines.

- Check the Availability of key Essential Medicines List.

\subsection{Previous Similar Studies}

A systematic review of the literature that studied the WHO drug core indicators for African region from 1995 up to 2015 that conducted by Ofori-Asenso et al., found that the prescribing indicators deviate significantly from the WHO reference targets [5]. Bashir and Siripen have assessed the general prescription pattern at primary health care centers in NHIF in Gezira, Sudan [6]. Sharif, et al. studied the pattern of drug prescription in private hospitals in Dubai [7]. The results of Primary health care physicians' prescribing patterns for children under five in Qassim, Saudi Arabia, was not at the optimal level of rational use, especially regarding the prescription of antibiotics as studied by Jahan et al. [8]. Antibiotics prescription at primary healthcare facilities in Addis Ababa, Ethiopia was assessed by Fikru and Dagmawit [9]. Other studies were carried out in Ethiopia also, by Muluken et al. to assess prescription patterns of drugs in Finote Selam and Motta District Hospital outpatient pharmacy [10], and in Northeast Ethiopia, and they found that the majority of indicators not met by WHO standards [11]. At Hawassa University Teaching and Referral Hospital in Southern Ethiopia, Anteneh conducted a descriptive study to determine the current prescribing practices there, and he found that there was a significant deviation from the standard recommended by WHO due to commonly overused and costly forms of drug [12]. The status in eastern Ethiopia is the same as mentioned before that the majority of WHO stated core drug use indicators were not met by the three hospitals included in the study conducted by Mekonnen [13]. The evidence of irrational drug prescription, especially in terms of injections, antibiotics, and poly-pharmacy was found in a different setting in Iran [14] [15]. In 
Ghana, a retrospective study using WHO core indicators were carried out [16], and other in Northern Ghana and again the indicators of prescribing did not comply with WHO standards [17]. Two studies were conducted by Muhammed Atif and others in two areas of Pakistan, Bahawalpur, and Punjab, and the results showed a remarkable deviation from the standard [18] [19]. In India Singh et al. made a prescription audit using the WHO-recommended core drug use indicators in a rural hospital of Delhi, and concluded that the urgent training is need for prescribers on writing rationally [20]. Tawfeeg et al. studied the drugs core indicators in the Kurdistan Region, and they found that the majority of indicators were higher than the suggested by WHO [21]. In Nepal, Nepal et al. studied the antibiotic prescribing pattern in public health facilities, and they found it higher compared with WHO guidelines [22]. In Namibia, the WHO indicators showed poor accuracy in assessing prescribing practices in Primary Health Care [23].

\section{Methods}

A cross-sectional, prospective, analytical study was conducted in 30 randomly selected out of 47 health facilities in (WHO recommend minimum 20 facilities as convenient sample), in outpatient medical services provided by general medical doctors in Wadmadani locality, Gezira state, central Sudan from 26 November 2017 to three December 2018. A total of 1800 random prescriptions from all selected health centers regarding both prescribing and patient care indicators (60 from each center) were prospectively selected (WHO recommend minimum 30 prescriptions from each facility as a convenient sample). For health facility indicators, an observation checklist was performed to ensure the availability of essential guidelines and key drugs in the stock. Three well-trained pharmacists were collected the data using the form sheet as described in the WHO manual [2]. Independent sample t-test was carried out to compare between direct health facility (services provided by NHIF) and indirect (services provided by $\mathrm{SMOH}$ ), for numerical indicators, while chi-square test was done for categorical indicators to test whether there was a statistical difference at the level of confidence of $95 \%$. The data were analyzed using the SPSS version 16.

\subsection{Prescribing Indicators}

The average number of drugs per encounter was calculated by dividing the total number of different drugs prescribed by the number of encounters surveyed. The percentage of drugs prescribed in generics was calculated by dividing the number of drugs prescribed by generic name by the total number of drugs prescribed, multiplied by 100 . The percentage of prescriptions with antibiotics was calculated by dividing the number of patient encounters with an antibiotic prescribed by the total number of encounters surveyed, multiplied by 100 . The Percentage of prescriptions with injections was calculated by dividing the number of patient encounters with injection by the total number of encounters surveyed, 
multiplied by 100. The prescription from EML was calculated as a percentage (dividing total prescribed drugs from EML by the total prescribed, multiply by 100) $[2]$.

\subsection{Patient Care Indicators}

The average consultation time was calculated by dividing the total time for a series of consultations, by the number of consultations. Average dispensing time was calculated by dividing the total time for dispensing drugs to a series of patients, by the number of encounters. The percentage of drugs actually dispensed was calculated by dividing the number of drugs actually dispensed at the health facility by the total number of drugs prescribed, multiplied by 100 . The percentage of drugs adequately labeled was calculated by dividing the number of drug packages containing at least drug name and strength and how frequent and length the drug should be taken, by the total number of drug packages dispensed, multiplied by 100. Patient's knowledge of correct dosage was calculated by dividing the number of patients who can adequately report the dosage schedule for all drugs, by the total number of patients interviewed, multiplied by 100 [2].

\subsection{Facility Indicators}

The availability of EML was checked by saying yes or no, per facility after observation. Key drugs availability was calculated by dividing the number of specified products actually in stock by the total number of drugs on the checklist, multiplied by $100[2]$.

\section{Area of the Study}

NHIF is a governmental scheme in Sudan aiming to achieve social protection through Universal Health Coverage (UHC) and mitigate the catastrophic expenditure (out of pocket). It was established in 1994, firstly implemented in Sinnar State followed by Khartoum and Gezira States in 1997. Then the rest of the states respectively.

Gezira is a central state in Sudan consisting of eight localities (total area of $25,549 \mathrm{Km}^{2}$ ), with an estimated population of five million. Wadmadin locality is the capital of the state with more than half a million population. It consists of five units. The distribution of selected health centers in the units of the locality was shown in Table 1.

\section{Results}

The results of frequencies, means, standard deviation, and independent t-test of prescribing, dispensing, and facility Drug core indicators of selected direct and indirect health centers in this present study were summarized in Tables 2-4 respectively. A Chi-square test was conducted only for the availability of the EML indicator because it is the only categorical variable. 
Table 1. Distribution of selected direct and indirect facility in units of the locality.

\begin{tabular}{cccccccc}
\hline \multirow{2}{*}{$\begin{array}{c}\text { Type of } \\
\text { Health } \\
\text { Center }\end{array}$} & \multicolumn{5}{c}{ Distribution of selected Health Centers in The locality } \\
\cline { 2 - 6 } & $\begin{array}{c}\text { East } \\
\text { area }\end{array}$ & $\begin{array}{c}\text { Central } \\
\text { market }\end{array}$ & $\begin{array}{c}\text { North/West } \\
\text { area }\end{array}$ & $\begin{array}{c}\text { Central } \\
\text { Madani }\end{array}$ & $\begin{array}{c}\text { Hantoub } \\
\text { Unit }\end{array}$ & $\begin{array}{c}\text { Alshabarga } \\
\text { Unit }\end{array}$ & Total/\% \\
\hline Direct* & 3 & 0 & 5 & 1 & 2 & 0 & $11(36.7)$ \\
Indirect** & 5 & 3 & 4 & 3 & 3 & 1 & $19(63.3)$ \\
Total/\% & $8(27)$ & $3(10)$ & $9(30)$ & $4(13)$ & $5(17)$ & $1(3)$ & $30(100)$ \\
\hline
\end{tabular}

${ }^{\star}$ Direct: services provided by NHIF ${ }^{* *}$ Indirect: services provided by State Ministry of Health.

Table 2. Prescribing drug core indicators results summary.

\begin{tabular}{cccccc}
\hline \multirow{2}{*}{$\begin{array}{c}\text { Indicators Assessed } \\
\text { N }\end{array}$} & \multicolumn{2}{c}{ Mean \pm Standard Deviation } & \multirow{2}{*}{$\boldsymbol{P}$ value } & Standard $^{*}$ \\
\cline { 2 - 5 } & Direct & Indirect & Overall & & \\
\hline Drugs per encounter & $2.9 \pm 0.7$ & $2.3 \pm 0.4$ & $2.5 \pm 0.6$ & 0.060 & 1.8 \\
Drugs prescribed by generic & $37.5 \pm 10.4$ & $48.0 \pm 15.0$ & $44.1 \pm 14.2$ & 0.049 & $100 \%$ \\
Encounters with an antibiotic & $47.9 \pm 22.5$ & $58.6 \pm 14.2$ & $54.7 \pm 18.0$ & 0.117 & $30 \%$ \\
Encounters with an Injections & $11.8 \pm 9.0$ & $12.2 \pm 9.7$ & $12.0 \pm 9.3$ & 0.819 & $20 \%$ \\
Drugs prescribed from EML & $97.5 \pm 2.5$ & $93.8 \pm 14.3$ & $95.2 \pm 11.5$ & 0.413 & $100 \%$ \\
\hline
\end{tabular}

${ }^{*}$ According to WHO drug core indicators. ${ }^{*}$ Essential Medicines List.

Table 3. Dispensing drug core indicators results summary.

\begin{tabular}{|c|c|c|c|c|c|}
\hline \multirow{2}{*}{ Indicators Assessed } & \multicolumn{3}{|c|}{ Mean \pm Standard Deviation } & \multirow{2}{*}{$P$ value } & \multirow{2}{*}{ Standard } \\
\hline & Direct & Indirect & Overall & & \\
\hline Average consultation time & $3.2 \pm 1.0$ & $2.7 \pm 0.6$ & $2.9 \pm 0.8$ & 0.141 & $10 \mathrm{Min}$ \\
\hline Average dispensing time & $116.6 \pm 53.6$ & $89.7 \pm 17.3$ & $99.5 \pm 36.8$ & 0.052 & $180 \mathrm{Sec}$ \\
\hline Drugs actually dispensed & $70.5 \pm 10.7$ & $73.6 \pm 18.6$ & $72.5 \% \pm 16.0 \%$ & 0.615 & $100 \%$ \\
\hline Drugs adequately labeled & $46.1 \pm 17.9$ & $50.8 \pm 18.3$ & $49.0 \% \pm 18.0 \%$ & 0.504 & $100 \%$ \\
\hline Patients' knowledge of correct dosage & $21.6 \pm 7.1$ & $23.1 \pm 7.5$ & $22.5 \% \pm 7.3 \%$ & 0.579 & $100 \%$ \\
\hline
\end{tabular}

Table 4. Facility specific drug core indicators results summary:

\begin{tabular}{|c|c|c|c|c|c|}
\hline \multirow{2}{*}{ Indicators Assessed } & \multicolumn{3}{|c|}{ Mean \pm Standard Deviation } & \multirow{2}{*}{$P$ value } & \multirow{2}{*}{ Standard } \\
\hline & Direct & Indirect & Overall & & \\
\hline Availability of copy of NHI-EML* & $72.7 \pm \mathrm{NA}$ & $63.2 \pm \mathrm{NA}$ & $66.7 \pm \mathrm{NA}$ & 0.592 & $100 \%$ \\
\hline Availability of key drugs & $71.4 \pm 10.0$ & $77.5 \pm 12.0$ & $75.3 \% \pm 11.6$ & 0.162 & $100 \%$ \\
\hline
\end{tabular}

${ }^{*}$ Chi-squired test for this categorical variable was done. NA: Not Available.

\section{Discussion}

\subsection{Prescribing Indicators}

In this present study, $95.2 \% \pm 11.5 \%$ of drugs were prescribed according to the NHIF-EML. When these results were compared to other studies, it found higher than Yousif and Siripen, 81.9\% [6], and in Ethiopia (66\% - 81\%) [9] [13]. In Pa- 
kistan, the range was found between (93\% - 98\%) [18] [19] and all the prescriptions within the list (100\%) in Ghana [16].

The mean of drugs per encounter was $2.5 \pm 0.6$, which is high according to the WHO standard (1.8). These results were found the same as obtained by Yousif study [6]in Gezira State, Sudan (2.5), and Dubai (2.2) [7]. And was high when compared with that found in Motta Hospital at Ethiopia (1.2) [10], south Ethiopia (1.9) [12], and east Ethiopia (2.34) [13], and lower than that obtained in Ghana (2.9) [16], Iran (3.14) [14], Pakistan (2.8 - 3.4) [18] [19], and Nigeria (6.11) [24].

The percentage of drugs prescribed by generic name was $44.1 \% \pm 14.2 \%$. This is near to Yousif study (46\%) [6], and lower than in Ethiopia (98\%) [10] [12], Iran (95\%) [14], Ghana (99.8\%) [16], and Pakistan (65\% - 71\%) [18] [19]. This lower result in this study may be attributed to the influence of local and international pharmaceutical companies on physician's decisions. The t-test for this indicator between direct (Mean $37.5 \pm$ SD10.4) and indirect (48.0 \pm 15.0) facilities showed significant differences (0.049). This indicates a strong alert for intervention in the direct facility.

Fifty-four percent \pm 18.0 of prescribed drugs as antibiotics, this is notably less than that reported in Ethiopia (56\% - 58\%) [9] [13], Yemen (60\%) [25] and higher than that in Dubai (21\%) [7], Ghana (36\%) [16], Iran (52\%) [14], and Pakistan (48\% - 51\%) [18] [19]. Overuse of antibiotics is one of the major factors in the emergence of resistant strains of bacterial pathogens. Over-prescription of antibiotics can lead to major problems in clinical practice.

The percentage of injectable medicines in this study was $12.0 \% \pm 9.3 \%$, and it was similar to the study conducted in NHIF, Gezira state [6], and was found to be lower than that in Pakistan (27\%) [18] [19], Iran (24\%) [14], Ethiopia (38\%) [10], and Nigeria (71.7\%) [24].

\subsection{Patient Care Indicators}

The result of the current study demonstrated that the average consultation time was $2.9 \pm 0.8$ minutes (ideal time $\geq 10$ minutes), such a short consultation time was measured also in Pakistan (1.2 - 2.2 minutes) [18] [19], and lower than time spent in Nigeria (4.3) [24]. According to WHO, insufficient consultation time leads to incomplete patient examination and later irrational therapy [1]. Consultation time within the ideal range is considered enough for proper history taking, complete physical examination, proper health education, and good physician-patient interaction. Short consultation time could be the consequence of an increased workload of the physicians at the healthcare center.

The study reported an average dispensing time of $99.5 \pm 36.8$ seconds (optimal value $\geq 180 \mathrm{Sec}$ ). This finding was accepted when compared to the findings from Pakistan (8 - 38 seconds) [18] [19]. Sufficient dispensing time is crucial for adequate labeling, and to give complete information about drug regimen, unwanted drug effects, and precautions of drugs. Insufficient information about therapy could lead to non-compliance and next adverse events. 
The percentage of medicines actually dispensed was $72.5 \% \pm 16.0 \%$ less than the ideal standard (100\%). This was lower than reported in Pakistan (90\% - 97\%) [18] [19]. The main reason involved in the low percentage of actually dispensed drugs could be the inadequate availability of drugs in the stock.

The percentage of medicines adequately labeled was $49.0 \% \pm 18.0 \%$. WHO recommends that dispensed drugs should be adequately labeled with respect to the patient's name, the dose of the drug, and regimen [1].

According to our findings, the patient's knowledge of the correct dose was $22.5 \% \pm 7.3 \%$ (less than the ideal value of $100 \%$ ). It was lower than that reported in Pakistan (61\% - 62\%) [18] [19]. Patient's knowledge about correct dosage is highly significant to avoid overuse and miss bused of medicines, and prevent adverse events that ultimately affect the patient's health. Limited drug-related knowledge of the patients may be associated with the increased workload of the healthcare providers, and poor understanding skills of the patients.

\subsection{Facility Specific Indicators}

The study revealed that all health centers providing the medical service to the NHIF had a deficit in a copy of NHIF-EML (66.7\%) which was far from the proposed norms (ideal value 100\%). A study from Pakistan [18] [19] reported $72 \%-82 \%$ was the percentage of the health sectors that had the medicine list copy.

The percentage of key drugs in the stock was $75.3 \% \pm 11.6 \%$ (optimal value is $100 \%)$. Shortage in the availability of key drugs might be associated with budgetary constraints, inadequate drug supply system, or poor inventory management of the responsible staff.

\section{Conclusion}

Most of the core drug use indicators were not met with the WHO standards. Interventions to decrease poly-pharmacy, and improve generic and antibiotics prescribing indicators were needed. The patient-to-healthcare cadre ratio should be revised to optimize consultation, and dispensing time. The availability of key drugs should be improved through an efficient supply chain to ensure effective treatment. The pharmacy cadre should be oriented and trained to improve patients' compliance. This description of core drug indicators will provide a road map for appropriate intervention toward a rational use of medicine in NHIF health centers, Gezira State Sudan in recent coming future.

\section{Ethical Approval}

Ethical approval from the National Health Insurance Fund (NHIF), Gezira State, Sudan was taken formally before conducting the research. Verbal consent was obtained from patients and health professionals before starting the interview.

\section{Acknowledgements}

The authors acknowledge National Health Insurance Fund, Gezira State for its 
logistic facilitation, and all members who participate in the study, data collection, and analysis.

\section{Funding}

This study was not funded by any organization.

\section{Conflicts of Interest}

The authors declare no conflicts of interest regarding the publication of this paper.

\section{References}

[1] World Health Organization (2009) Medicines Use in Primary Care in Developing and Transitional Countries: Fact Book Summarizing Results from Studies Reported between 1990 and 2006. World Health Organization, Geneva.

[2] World Health Organization (1993) How to Investigate Drug Use in Health Facilities: Selected Drug Use Indicators. World Health Organization, Geneva.

[3] World Health Organization (2002) Promoting Rational Use of Medicines: Core Components. World Health Organization, Geneva.

[4] Directorate of Planning and Statistics, Annual Statistical Report (2018) National Health Insurance Fund, Gezira State Branch, Sudan.

[5] Ofori-Asenso, R., Brhlikova, P. and Pollock, A.M. (2016) Prescribing Indicators at Primary Health Care Centers within the WHO African Region: A Systematic Analysis (1995-2015). BMC Public Health, 16, Article No. 724. https://doi.org/10.1186/s12889-016-3428-8

[6] Yousif, B.M.E. and Supakankunti, S. (2016) General Practitioners' Prescribing Patterns at Primary Healthcare Centers in National Health Insurance, Gezira, Sudan. Drugs-Real World Outcomes, 3, 327-332. https://doi.org/10.1007/s40801-016-0087-0

[7] Sharif, S., Al-Shaqra, M., Hajjar, H., Shamout, A. and Wess, L. (2008) Patterns of Drug Prescribing in a Hospital in Dubai, United Arab Emirates. Libyan Journal of Medicine, 3, 10-12. https://doi.org/10.3402/ljm.v3i1.4744

[8] Jahan, S., Al-Saigul, A.M. and Hamdelsseed, S.A. (2019) Primary Health Care Physicians' Prescribing Patterns for Children under Five in Qassim, Saudi Arabia. Primary Health Care Research \& Development, 20, Article No. 89. https://doi.org/10.1017/S1463423619000148

[9] Worku, F. and Tewahido, D. (2018) Retrospective Assessment of Antibiotics Prescribing at Public Primary Healthcare Facilities in Addis Ababa, Ethiopia. Interdisciplinary Perspectives on Infectious Diseases, 2018, Article ID: 4323769. https://doi.org/10.1155/2018/4323769

[10] Wubetu, M., Derebe, D., Mulaw, T., Yimer, T. and Hailu, G. (2018) Assessment of Drug Prescription Pattern in Two District Hospitals, Northwest Ethiopia. Journal of Health Education Research and Development, 6, Article No. 246. https://doi.org/10.4172/2380-5439.1000246

[11] Mamo, D.B. and Alemu, B.K. (2020) Rational Drug-Use Evaluation Based on World Health Organization Core Drug-Use Indicators in a Tertiary Referral Hospital, Northeast Ethiopia: A Cross-Sectional Study. Drug, Healthcare and Patient Safety, 12, 15-21. https://doi.org/10.2147/DHPS.S237021

[12] Desalegn, A.A. (2013) Assessment of Drug Use Pattern Using WHO Prescribing 
Indicators at Hawassa University Teaching and Referral Hospital, South Ethiopia: A Cross-Sectional Study. BMC Health Services Research, 13, Article No. 170. https://doi.org/10.1186/1472-6963-13-170

[13] Sisay, M., Mengistu, G., Molla, B., Amare, F. and Gabriel, T. (2017) Evaluation of Rational Drug Use Based on World Health Organization Core Drug Use Indicators in Selected Public Hospitals of Eastern Ethiopia: A Cross Sectional Study. BMC Health Services Research, 17, Article No. 161. https://doi.org/10.1186/s12913-017-2097-3

[14] Ahmadi, F. and Zarei, E. (2017) Prescribing Patterns of Rural Family Physicians: A Study in Kermanshah Province, Iran. BMC Public Health, 17, Article No. 908. https://doi.org/10.1186/s12889-017-4932-1

[15] Zarei, E. and Peyvandi, H. (2020) Determination of Drug Prescription Pattern for Outpatients Covered by Social Security Organization in Semnan Province and Compare It with the WHO Standards. Depiction of Health, 10, 248-258.

[16] Apanga, S., Chirawurah, D., Kudiabor, C., Adda, J., Adoesom, J.A. and Punguyire, D. (2014) Evaluation of Drug Prescribing Pattern under the National Health Insurance Scheme in Rural Ghana. International Journal of Pharmaceutical Sciences and Research, 5, 2193-2198.

[17] Mohammed, B.S. and Tiah, S.A. (2019) Medicines Prescribing Pattern in Northern Ghana: Does It Comply with WHO Recommendations for Prescribing Indicators? African Journal of Pharmacy and Pharmacology, 13, 70-75. https://doi.org/10.5897/AJPP2018.4981

[18] Atif, M., Sarwar, M.R., Azeem, M., Naz, M., Amir, S. and Nazir, K. (2016) Assessment of Core Drug Use Indicators Using WHO/INRUD Methodology at Primary Healthcare Centers in Bahawalpur, Pakistan. BMC Health Services Research, 16, Article No. 684. https://doi.org/10.1186/s12913-016-1932-2

[19] Atif, M., Sarwar, M.R., Azeem, M., Umer, D., Rauf, A., Rasool, A., Ahsan, M. and Scahill, S. (2016) Assessment of WHO/INRUD Core drug Use Indicators in Two Tertiary Care Hospitals of Bahawalpur, Punjab, Pakistan. Journal of Pharmaceutical Policy and Practice, 9, Article No. 27. https://doi.org/10.1186/s40545-016-0076-4

[20] Singh, T., Banerjee, B., Garg, S. and Sharma, S., (2019) A Prescription Audit Using the World Health Organization-Recommended Core Drug Use Indicators in a Rural Hospital of Delhi. Journal of Education and Health Promotion, 8, Article No. 37.

[21] Tawfeeq, R., Tahssen Obied, T. and Ommar, A. (2019) Assessment of Prescriptions Patterns and Drug Use across the Kurdistan Region. Journal of Pharmaceutical Care, 7, 26-30. https://doi.org/10.18502/jpc.v7i1-2.1619

[22] Anant, N., Delia, H., Suzanne, R. and Selvey, L.A. (2020) Analysis of Patterns of Antibiotic Prescribing in Public Health Facilities in Nepal. The Journal of Infection in Developing Countries, 14, 18-27. https://doi.org/10.3855/jidc.11817

[23] Niaz, Q., Godman, B., Massele, A., Campbell, S., Kurdi, A., Kagoya, H. and Kibuule, D. (2019) Validity of World Health Organisation Prescribing Indicators in Namibia's Primary Healthcare: Findings and Implications. International Journal for Quality in Health Care, 31, 338-345. https://doi.org/10.1093/intqhc/mzy172

[24] Babalola, C., Awoleye, S., Akinyemi, J. and Kotila, O., (2011) Evaluation of Prescription Pattern in Osun State (Southwest) Nigeria. Journal of Public Health and Epidemiology, 3, 94-98.

[25] Bashrahil, K. (2010) Indicators of Rational Drug Use and Health Services in Hadramout, Yemen. Eastern Mediterranean Health Journal, 16, 151-155.

https://doi.org/10.26719/2010.16.2.151 